\title{
ZERO VISCOSITY LIMIT OF THE OSEEN EQUATIONS IN A CHANNEL*
}

\author{
MARIA CARMELA LOMBARDO ${ }^{\dagger}$ AND MARCO SAMMARTINO ${ }^{\dagger}$
}

\begin{abstract}
Oseen equations in the channel are considered. We give an explicit solution formula in terms of the inverse heat operators and of projection operators. This solution formula is used for the analysis of the behavior of the Oseen equations in the zero viscosity limit. We prove that the solution of Oseen equations converges in $W^{1,2}$ to the solution of the linearized Euler equations outside the boundary layer and to the solution of the linearized Prandtl equations inside the boundary layer.
\end{abstract}

Key words. Oseen equations, solution formula, zero viscosity limit, boundary layer, asymptotic expansion

AMS subject classifications. 35Q30, 76D07, 76D10, 35C20

PII. S0036141000372015

1. Introduction. In this paper we shall be concerned with the zero viscosity limit of the time dependent incompressible Oseen type equations in a two-dimensional channel.

Oseen equations are a simplified mathematical model in treating incompressible viscous fluids and their study should shed some light on the much more complicated physical phenomena described by the nonlinear Navier-Stokes equations.

The system we shall be concerned with is the following:

$$
\begin{aligned}
\left(\partial_{t}-\nu \Delta+U \partial_{x}\right) \boldsymbol{u}+\boldsymbol{\nabla} p & =\boldsymbol{f}, \\
\boldsymbol{\nabla} \cdot \boldsymbol{u} & =0, \\
\gamma^{-} \boldsymbol{u} & =\mathbf{g}^{-}, \\
\gamma^{+} \boldsymbol{u} & =\mathbf{g}^{+}, \\
\boldsymbol{u}(t=0) & =\boldsymbol{u}_{0} .
\end{aligned}
$$

In the above equations $\boldsymbol{u}=(u(x, y, t), v(x, y, t))$ is the unknown vector field, $p$ is the unknown pressure, $x \in \mathbb{R}$ denotes the tangential variable, $-1 \leq y \leq 1$ is the normal variable, $t \geq 0$ is the time, $\gamma$ is the trace operator, i.e., $\gamma^{-} \boldsymbol{u}=\boldsymbol{u}(x,-1, t)$ and $\gamma^{+} \boldsymbol{u}=\boldsymbol{u}(x, 1, t), \nu$ is the viscosity coefficient, and $\mathbf{g}^{-}, \mathbf{g}^{+}$, and $\boldsymbol{u}_{0}$ are the boundary and the initial data, respectively.

Equation (1.1) is obtained by linearizing the Navier-Stokes equation around the velocity flow $(U, 0)$, where $U$ is assumed to be constant. Equation (1.2) is the incompressibility condition. Equations (1.3) and (1.4) are the boundary conditions, while (1.5) is the initial condition.

The analysis of the zero viscosity limit of (1.1)-(1.5) (with homogeneous boundary data $\mathbf{g}^{+}=\mathbf{g}^{-}=0$ ) was performed by Temam and Wang in [4], [5], and [6]. In [6] the authors considered the more general case of the three-dimensional Navier-Stokes equations linearized around the flow $\left(U_{1}(z), U_{2}(z), 0\right)$. Without explicitly solving the

\footnotetext{
* Received by the editors May 3, 2000; accepted for publication (in revised form) February 22, 2001; published electronically July 19, 2001.

http://www.siam.org/journals/sima/33-2/37201.html

${ }^{\dagger}$ Dipartimento di Matematica, University of Palermo, Via Archirafi 34, 90123 Palermo, Italy (lombardo@dipmat.math.unipa.it,marco@dipmat.math.unipa.it). The research of the first author was partially supported by the INDAM. The research of the second author was partially supported by the GNFM-INDAM.
} 
equations and under the assumption of sufficient smoothness of the initial data, they proved that the solution can be decomposed in the following form:

$$
\boldsymbol{u}=\boldsymbol{u}^{0}+\tilde{\boldsymbol{\theta}}^{\varepsilon}+\nu^{1 / 4} \boldsymbol{w} \quad \text { with } \quad \boldsymbol{w} \in L^{2}\left([0, T] ;\left(W^{1,2}(\Omega)\right)^{3}\right),
$$

where $\boldsymbol{u}^{0}$ is the inviscid solution and $\tilde{\boldsymbol{\theta}}^{\varepsilon}$ is a corrector which takes into account the mismatch of the boundary conditions between the inviscid part and the overall solution.

In this paper we shall write the solution of (1.1)-(1.5) as the sum of an inviscid part, two Prandtl parts, and an error term, i.e.,

$$
\boldsymbol{u}=\boldsymbol{u}^{E}+\boldsymbol{u}^{P(-)}+\boldsymbol{u}^{P(+)}+\nu^{1 / 2} \boldsymbol{w} .
$$

In the above decomposition $\boldsymbol{u}^{E}$ is the inviscid (Euler) part describing the flow away from boundaries; $\boldsymbol{u}^{P(-)}$ and $\boldsymbol{u}^{P(+)}$ are the Prandtl parts describing the flow close to the two boundaries and decaying exponentially away from the two boundaries. Our main result is the following theorem.

THEOREM 1.1 (informal statement). Let $\boldsymbol{u}$ be the solution of (1.1)-(1.6). If the initial data $\boldsymbol{u}_{0}$ is in $H^{4}$, and if the boundary data $\boldsymbol{g}^{ \pm}$are sufficiently regular and are such that the normal components are integrable (in the sense specified by Definition 2.3 below), then

$$
\nu^{1 / 4} \boldsymbol{w} \in L^{\infty}\left([0, T] ; W^{1,2}\right)
$$

with norm independent of $\nu$.

Therefore the main advantage with respect to the results of $[6]$ is that here we prove that the norm of the correction term is $O\left(\nu^{1 / 4}\right)$ in $L^{\infty}\left([0, T] ; W^{1,2}\right)$, while there an analogous estimate is proved with a nondivergence-free boundary layer corrector. We also allow nonzero boundary data.

We shall achieve this result through the explicit solution formula for the Oseen equation. This explicit representation formula is, we believe, of independent interest.

The paper is organized as follows: in section 2 we shall introduce the functional setting. In section 3 the convection-diffusion operators in the channel are defined and we shall give some estimate in the appropriate function spaces. In section 4 we shall solve the Oseen equations with boundary data, zero initial data, and zero source term. The solution will be written in the form of an infinite series: the norm of the generic term of the series will be shown to be exponentially decaying. Convergence of the series will follow and the norm of the solution will be proved to be bounded in terms of the norm of the boundary data. In section 5 the complete Oseen system is solved introducing the projection operator onto the divergence-free function space and estimates of the solution in terms of the data are given. In section 6 we shall finally analyze the vanishing viscosity limit of the Oseen equations. The main results of this paper are formally stated in Theorem 6.5 and Corollary 6.1 .

2. The function spaces. In this section we define some function spaces we shall be using throughout the paper. We introduce the notation $\boldsymbol{\Omega} \equiv \mathbb{R} \times[-1,1]$. Here and in the rest of the paper $l \geq 2$.

DEFINITION 2.1. $H^{l}(\mathbb{R})$ is the set of all functions $f(x)$ such that

(i) $\frac{d^{j}}{d x^{j}} f \in L^{2}(\mathbb{R})$, where $j \leq l$.

We shall denote the usual norm in $H^{l}(\mathbb{R})$ with $|f|_{l}$. 
Definition 2.2. $H_{T}^{\prime l}$ is the set of all functions $f(x, t)$ such that

(i) $\partial_{t}^{j} f(x, t) \in L^{\infty}\left([0, T], H^{l-j}(\mathbb{R})\right)$ and $j \leq l$.

The norm of $f \in H_{T}^{\prime l}$ is given by

$$
|f|_{l, T}=\sum_{j_{1}+j_{2} \leq l} \sup _{0 \leq t \leq T}\left\|\partial_{t}^{j_{1}} \partial_{x}^{j_{2}} f(\cdot, t)\right\|_{L^{2}(\mathbb{R})} .
$$

Definition 2.3. $\boldsymbol{H}_{T}^{\prime l}$ is the set of all functions $\boldsymbol{f}=\left(f_{\tau}, f_{N}\right)$ such that

(i) $f_{\tau} \in H_{T}^{\prime l}$;

(ii) $|\xi|^{-1} f_{N} \in H_{T}^{\prime l+1}$.

The norm of $\boldsymbol{f} \in \boldsymbol{H}_{T}^{\prime l}$ is given by

$$
|f|_{l, T}=\left|f_{\tau}\right|_{l, T}+\left.\left.|| \xi\right|^{-1} f_{N}\right|_{l, T} .
$$

In the above definition $\xi$ is the dual Fourier variable of $x$, and $|\xi|^{-1}$ has to be understood as a pseudodifferential operator. The space $\boldsymbol{H}_{T}^{\prime l}$ is the space to which all boundary data we shall deal with in the rest of the paper will belong. The hypothesis on the normal component is an integrability hypothesis.

DeFINITION 2.4. $H^{l}$ is the set of all functions $f(x, y)$ such that

(i) $\partial_{x}^{i} \partial_{y}^{j} f(x, y) \in L^{2}(\boldsymbol{\Omega})$ for $i+j \leq l$.

The norm of $f$ is given by

$$
|f|_{l}=\sum_{i+j \leq l}\left\|\partial_{x}^{i} \partial_{y}^{j} f(\cdot, \cdot)\right\|_{L^{2}(\boldsymbol{\Omega})} .
$$

DEFINITION 2.5.

$H_{T}^{l}$ is the set of all functions $f(x, y, t)$ such that

- $\partial_{t}^{j} f(x, y, t) \in L^{\infty}\left([0, T], H^{l-j}\right)$ for $j \leq l$.

The norm of $f \in H_{T}^{l}$ is given by

$$
|f|_{l, T}=\sum_{j_{1}+j_{2}+j_{3} \leq l} \sup _{0 \leq t \leq T}\left\|\partial_{t}^{j_{1}} \partial_{x}^{j_{2}} \partial_{y}^{j_{3}} f(\cdot, \cdot, t)\right\|_{L^{2}(\boldsymbol{\Omega})} .
$$

All the above spaces are the natural ambient spaces for the Euler equations. We now introduce the ambient spaces for Prandtl equations. All the functions belonging to these spaces depend on the normal scaled variable $Y=y / \varepsilon$. We require differentiability with respect to this variable only up to the second order. We first introduce the spaces $K^{l, \mu(+)}$ and $K^{l, \mu(-)}$. The functions in these spaces are defined in the half plane $Y \leq 1 / \varepsilon$ and $Y \geq-1 / \varepsilon$, respectively, and decay exponentially fast away from $Y=1 / \varepsilon$ and $Y=-1 / \varepsilon$, respectively. In what follows $\mu>0$.

Definition 2.6. $K^{l, \mu( \pm)}$ is the set of all functions $f^{ \pm}(x, Y)$ defined for $-\infty<$ $\pm Y \leq 1 / \varepsilon$, and such that

(i) $\partial_{x}^{j_{1}} \partial_{Y}^{j_{2}} f^{ \pm}(x, Y) \in L^{2}(\mathbb{R})$, with $j_{2} \leq 2$ and $j_{1}+j_{2} \leq l$, $\leq l$.

(ii) $\sup _{-\infty< \pm Y \leq \frac{1}{\varepsilon}} e^{\mu\left(\frac{1}{\varepsilon} \mp Y\right)}\left\|\partial_{x}^{j_{1}} \partial_{Y}^{j_{2}} f^{ \pm}(\cdot, Y)\right\|_{L^{2}}<\infty$, where $j_{2} \leq 2$ and $j_{1}+j_{2}$

The norm is given by

$$
|f|_{l, \mu( \pm)}=\sum_{j_{2} \leq 2} \sum_{j_{1}+j_{2} \leq l} \sup _{-\infty< \pm Y \leq \frac{1}{\varepsilon}} e^{\mu\left(\frac{1}{\varepsilon} \mp Y\right)}\left\|\partial_{x}^{j_{1}} \partial_{Y}^{j_{2}} f^{ \pm}(\cdot, Y)\right\|_{L^{2}}
$$


Definition 2.7. $K^{l, \mu}$ is the set of functions $f(x, Y)$, defined for $-1 / \varepsilon \leq Y \leq$ $1 / \varepsilon$, such that

$$
f=f^{+}+f^{-},
$$

where $f^{+}$and $f^{-}$are restrictions to $-1 / \varepsilon \leq Y \leq 1 / \varepsilon$ of functions in $K^{l, \mu(+)}$ and $K^{l, \mu(-)}$, respectively. The norm is given by

$|f|_{l, \mu}=\sum_{j_{2} \leq 2} \sum_{j_{1}+j_{2} \leq l}\left[\sup _{0<Y \leq \frac{1}{\varepsilon}} e^{\mu\left(\frac{1}{\varepsilon}-Y\right)}\left\|\partial_{x}^{j_{1}} \partial_{Y}^{j_{2}} f(\cdot, Y)\right\|_{L^{2}}+\sup _{-\frac{1}{\varepsilon}<Y \leq 0} e^{\mu\left(\frac{1}{\varepsilon}+Y\right)}\left\|\partial_{x}^{j_{1}} \partial_{Y}^{j_{2}} f(\cdot, Y)\right\|_{L^{2}}\right]$.

We now introduce the dependence on time. We require differentiability with respect to time only up to the first order: one time derivative is equivalent to two space derivatives.

DEFINITION 2.8.

$K_{T}^{l, \mu( \pm)}$ is the set of all functions $f^{ \pm}(x, Y, t)$ such that

(i) $f \in L^{\infty}\left([0, T], K^{l, \mu( \pm)}\right)$,

(ii) $\partial_{t} \partial_{x}^{j} f \in L^{\infty}\left([0, T], K^{0, \mu( \pm)}\right)$ with $j \leq l-2$.

The norm is given by

$$
\begin{aligned}
|f|_{l, \mu, T( \pm)}= & \sum_{0 \leq j_{2} \leq 2} \sum_{j_{1} \leq l-2} \sup _{0 \leq t \leq T} \sup _{-\infty< \pm Y \leq \frac{1}{\varepsilon}} e^{\mu\left(\frac{1}{\varepsilon} \mp Y\right)}\left\|\partial_{x}^{j_{1}} \partial_{Y}^{j_{2}} f^{ \pm}(\cdot, Y, t)\right\|_{L^{2}} \\
& +\sum_{j \leq l-2} \sup _{0 \leq t \leq T} \sup _{-\infty< \pm Y \leq \frac{1}{\varepsilon}} e^{\mu\left(\frac{1}{\varepsilon} \mp Y\right)}\left\|\partial_{t} \partial_{x}^{j} f^{ \pm}(\cdot, Y, t)\right\|_{L^{2}} .
\end{aligned}
$$

Definition 2.9. $K_{T}^{l, \mu}$ is the set of functions $f(x, Y, t)$, defined for $-1 / \varepsilon \leq Y \leq$ $1 / \varepsilon$, such that

$$
f=f^{+}+f^{-},
$$

where $f^{+}$and $f^{-}$are restrictions to $-1 / \varepsilon \leq Y \leq 1 / \varepsilon$ of functions in $K_{T}^{l, \mu(+)}$ and $K_{T}^{l, \mu(-)}$, respectively. The norm is given by

$$
\begin{aligned}
|f|_{l, \mu, T}= & \sum_{0 \leq j_{2} \leq 2} \sum_{j_{1} \leq l-2} \sup _{0 \leq t \leq T} \sup _{0<Y \leq \frac{1}{\varepsilon}} e^{\mu\left(\frac{1}{\varepsilon}-Y\right)}\left\|\partial_{x}^{j_{1}} \partial_{Y}^{j_{2}} f(\cdot, Y, t)\right\|_{L^{2}} \\
& +\sum_{j \leq l-2} \sup _{0 \leq t \leq T} \sup _{0<Y \leq \frac{1}{\varepsilon}} e^{\mu\left(\frac{1}{\varepsilon}-Y\right)}\left\|\partial_{t} \partial_{x}^{j} f(\cdot, Y, t)\right\|_{L^{2}} \\
& +\sum_{0 \leq j_{2} \leq 2} \sum_{j_{1} \leq l-2} \sup _{0 \leq t \leq T} \sup _{-\frac{1}{\varepsilon}<Y \leq 0} e^{\mu\left(\frac{1}{\varepsilon}+Y\right)}\left\|\partial_{x}^{j_{1}} \partial_{Y}^{j_{2}} f(\cdot, Y, t)\right\|_{L^{2}} \\
& +\sum_{j \leq l-2} \sup _{0 \leq t \leq T} \sup _{-\frac{1}{\varepsilon}<Y \leq 0} e^{\mu\left(\frac{1}{\varepsilon}+Y\right)}\left\|\partial_{t} \partial_{x}^{j} f(\cdot, Y, t)\right\|_{L^{2}} .
\end{aligned}
$$

We now introduce the ambient spaces for the error equation. All functions belonging to the following spaces are functions $L^{2}$ with respect to both tangential and normal variables. Notice that, due to the presence in the error equation of the rapidly varying terms arising from the Prandtl solution, the solution of the error equation will have a fast dependence on $y$. Therefore, in the following spaces, all the derivatives of order $j$ with respect to $y$ are weighted with $\varepsilon^{j} \equiv \nu^{j / 2}$. 
DEFINITION 2.10. $L^{l}$ is the set of all functions $f(x, y)$ such that

(i) $\partial_{x}^{j_{1}} \varepsilon^{j_{2}} \partial_{y}^{j_{2}} f \in L^{2}(\boldsymbol{\Omega})$ with $j_{2} \leq 2$ and $j_{1}+j_{2} \leq l$.

The norm of $f \in L^{l}$ is given by

$$
\|f\|_{l}=\sum_{j_{1} \leq l}\left\|\partial_{x}^{j_{1}} f\right\|_{L^{2}(\boldsymbol{\Omega})}+\sum_{0 \leq j_{2} \leq 2} \sum_{j_{1} \leq l-2}\left\|\partial_{x}^{j_{1}} \varepsilon^{j_{2}} \partial_{y}^{j_{2}} f\right\|_{L^{2}(\boldsymbol{\Omega})} .
$$

DEFinition 2.11. $L_{T}^{\prime l}$ is the set of all functions $f(x, t)$ such that

(i) $\partial_{t} \partial_{x}^{j} f \in L^{\infty}\left([0, T], H^{l}(\mathbb{R})\right)$ for $j \leq l-2$.

The norm of $f \in L_{T}^{\prime l}$ is given by

$$
\|f\|_{l, T}=\sum_{j \leq l-2} \sup _{0 \leq t \leq T}\left\|\partial_{t} \partial_{x}^{j} f(\cdot, t)\right\|_{L^{2}(\boldsymbol{\Omega})} .
$$

DEFINITION 2.12. $L_{T}^{l}$ is the set of all functions $f(x, y, t)$ such that

(i) $f \in L^{\infty}\left([0, T], L^{l}\right)$,

(ii) $\partial_{t} \partial_{x}^{j} f \in L^{\infty}\left([0, T], L^{0}\right)$ with $j \leq l-2$.

The norm of $f \in L_{T}^{l}$ is given by

$\|f\|_{l, T}=\sum_{0 \leq j_{2} \leq 2} \sum_{j_{1} \leq l-2} \sup _{0 \leq t \leq T}\left\|\partial_{x}^{j_{1}} \varepsilon^{j_{2}} \partial_{y}^{j_{2}} f(\cdot, \cdot, t)\right\|_{L^{2}(\boldsymbol{\Omega})}+\sum_{j \leq l-2} \sup _{0 \leq t \leq T}\left\|\partial_{t} \partial_{x}^{j} f(\cdot, \cdot, t)\right\|_{L^{2}(\boldsymbol{\Omega})}$.

3. The convection-diffusion operators in the channel. In this section we shall give an explicit representation of the convection-diffusion operators $F_{0}, F_{1}$, and $F_{2}$. These operators solve the convection-diffusion equation in the channel with initial data, boundary data, and source term, respectively. Our explicit representation is given in terms of the action on the Fourier transform in the tangential variable. These operators will be used in the construction of the explicit solution of the Oseen equations. In what follows $\xi$ will always denote the dual Fourier variable of $x$ :

$$
\hat{f}\left(\xi^{\prime}\right)=\frac{1}{(2 \pi)^{1 / 2}} \int_{-\infty}^{+\infty} d x f(x) e^{-i \xi^{\prime} x} .
$$

In the rest of the paper we shall not distinguish between a function and its Fourier transform.

The convection-diffusion equation in the channel writes as follows:

$$
\begin{aligned}
\left(\partial_{t}-\nu \Delta+U \partial_{x}\right) u & =f, \\
\gamma^{+} u & =g^{+}, \\
\gamma^{-} u & =g^{-}, \\
\left.u\right|_{t=0} & =u_{0} .
\end{aligned}
$$

The operator $F_{0}$ solves the above equations with initial data and zero boundary data and zero source term. If we now introduce the $\theta$-function (see [8, Chapter V]),

$$
\theta(y, t)=\sum_{n=-\infty}^{\infty} k(y+4 n, t), \quad-\infty<y<\infty
$$

where

$$
k(y, t)=\frac{e^{-y^{2} / 4 \nu t}}{(4 \pi \nu t)^{1 / 2}}
$$


one can easily see that the explicit expression of $F_{0}$ is given by

$$
F_{0} u_{0}=e^{-\left[\nu \xi^{2}+i \xi U\right] t} \int_{-1}^{+1} d y^{\prime}\left[\theta\left(y-y^{\prime}, t\right)-\theta\left(y+y^{\prime}+2, t\right)\right] u_{0}\left(\xi, y^{\prime}\right) .
$$

The operator $F_{1}$ solves the convection-diffusion equations in the channel with boundary data, zero initial data, and zero source term: If we introduce the $\varphi$-function (see [8])

$$
\varphi(y, t)=\sum_{n=-\infty}^{+\infty} h(y+4 n, t), \quad-\infty<y<\infty
$$

where

$$
h(y, t)=\frac{y}{t} \frac{e^{-y^{2} /(4 \nu t)}}{\sqrt{4 \pi t \nu}},
$$

one can easily verify that the operator $F_{1}$ has the following expression:

$$
\begin{aligned}
F_{1}\left(g^{+}, g^{-}\right) & =\int_{0}^{t} d s e^{-\left(\nu \xi^{2}+i \xi^{\prime} U\right)(t-s)} \varphi(y+1, t-s) g^{-}\left(\xi^{\prime}, s\right) \\
& +\int_{0}^{t} d s e^{-\left(\nu \xi^{\prime 2}+i \xi^{\prime} U\right)(t-s)} \varphi(1-y, t-s) g^{+}\left(\xi^{\prime}, s\right) .
\end{aligned}
$$

Finally, the operator $F_{2}$ solves the convection-diffusion equations with source term, zero initial data, and zero boundary data. One can give the following explicit expression for $F_{2}$ :

$$
F_{2} f=\int_{0}^{t} d s e^{-\left[\nu \xi^{2}+i \xi U\right](t-s)} \int_{-1}^{+1} d y^{\prime}\left[\theta\left(y-y^{\prime}, t-s\right)-\theta\left(y+y^{\prime}+2, t-s\right)\right] f\left(\xi, y^{\prime}, s\right) .
$$

We now give some estimates on the above operators. In the propositions below $c$ is a constant that does not depend on $\nu$. The proofs of the proposition below are standard and can be achieved using the explicit representation formulas (3.5), (3.6), and (3.7) and the same arguments of [3], where the heat operators in the half plane are considered.

Proposition 3.1. Let $u_{0} \in L^{l}$ with $\gamma^{ \pm} u_{0}=0$. Then $F_{0} u_{0} \in L_{T}^{l}$ and the following estimate holds:

$$
\left\|F_{0} u_{0}\right\|_{l, T} \leq c\left\|u_{0}\right\|_{l} .
$$

Proposition 3.2. Let $g^{+} \in L_{T}^{\prime l}, g^{-} \in L_{T}^{\prime l}$ satisfy the compatibility condition $g^{+}(t=0)=g^{-}(t=0)=0$. Then $F_{1}\left(g^{+}, g^{-}\right) \in L_{T}^{l}$ and the following estimate holds:

$$
\left\|F_{1}\left(g^{+}, g^{-}\right)\right\|_{l, T} \leq c\left[\left\|g^{+}\right\|_{l, T}+\left\|g^{-}\right\|_{l, T}\right] .
$$

Proposition 3.3. Let $g^{+} \in L_{T}^{\prime l}, g^{-} \in L_{T}^{\prime l}$, and $g^{+}(t=0)=g^{-}(t=0)=0$; then $F_{1}\left(g^{+}, g^{-}\right) \in K_{T}^{l, \mu}$ and the following estimate holds:

$$
\left|F_{1}\left(g^{+}, g^{-}\right)\right|_{l, \mu, T} \leq c\left[\left\|g^{+}\right\|_{l, T}+\left\|g^{-}\right\|_{l, T}\right] .
$$

Proposition 3.4. Let $f \in L_{T}^{l}$; then $F_{2} f \in L_{T}^{l}$ and the following estimate holds:

$$
\left\|F_{2} f\right\|_{l, T} \leq c\|f\|_{l, T} \text {. }
$$


The following estimate on the operator $F_{2}$ says that, if the source term is of the same order of the square root of $\nu$, then one can use the heat kernel to increase the regularity of the solution.

Proposition 3.5. Let $f=\sqrt{\nu} h$ with $h \in L_{T}^{l-1}$; then $F_{2} f \in L_{T}^{l}$ and the following estimate holds:

$$
\left\|F_{2} f\right\|_{l, T} \leq c\|h\|_{l-1, T} .
$$

4. The Oseen equations with boundary data: The explicit solution. In this section we shall derive the explicit solution of the Oseen equations in the channel with boundary data, zero source term, and zero initial data, namely,

$$
\begin{aligned}
\left(\partial_{t}-\nu \Delta+U \partial_{x}\right) \boldsymbol{u}+\nabla p & =0, \\
\nabla \cdot \boldsymbol{u} & =0, \\
\gamma^{-} \boldsymbol{u} & =\mathbf{g}^{-}, \\
\gamma^{+} \boldsymbol{u} & =\mathbf{g}^{+}, \\
\left.\boldsymbol{u}\right|_{t=0} & =0,
\end{aligned}
$$

where $\mathbf{g}^{+}=\left(g_{\tau}^{+}, g_{N}^{+}\right)$and $\mathbf{g}^{-}=\left(g_{\tau}^{-}, g_{N}^{-}\right)$.

We shall express the solution of the above equations as a series. The $n$th $(n$ even) term of the series solves Oseen equations in the channel with the right (in the sense that it cancels the boundary datum created at $y=-1$ by the $(n-1)$ th term) boundary condition at $y=-1$. On the other hand, the $n$th term of the series creates a boundary datum at $y=+1$, which will be canceled by the $(n+1)$ th term, which therefore has the right boundary condition at $y=+1$. Of course the main concern will be to show that this series is convergent. In fact we shall prove that, for a time $T_{\alpha}$, this series is geometric with parameter $\alpha<1$. Our representation for the solution of (4.1)-(4.5) will therefore be valid only up to time $T_{\alpha}$. Notice, however, that $T_{\alpha} \rightarrow \infty$ when $\nu \rightarrow 0$.

The plan of the section is the following. First we introduce some pseudodifferential operators: (1) the operators $U^{ \pm}$(that we call the Ukai operators) that are projection operators, $(2)$ the operator $\mathcal{L}$ solving Dirichlet problem in the strip, and (3) the projection-convection-diffusion operators $\mathcal{M}^{+}$and $\mathcal{M}^{-}$. All these operators are the ingredients to construct, in section 4.4, the operators $\mathcal{O}^{-}$and $\mathcal{O}^{+}$. These are operators that solve Oseen equations with the right boundary condition only at $y=-1$ and at $y=+1$, respectively. Finally, through these operators, in section 4.5 we construct the explicit solution formula.

4.1. The Ukai operators. Here we define the following operators which are a modification of the operator $U$ introduced in [7]:

$$
\begin{aligned}
& U^{-} f=\int_{-1}^{y} d y^{\prime} e^{-|\xi|\left(y-y^{\prime}\right)} f\left(y^{\prime}, \xi\right), \\
& U^{+} f=-\int_{y}^{1} d y^{\prime} e^{|\xi|\left(y-y^{\prime}\right)} f\left(y^{\prime}, \xi\right) .
\end{aligned}
$$

These operators solve the problems

$$
\begin{aligned}
\left(\partial_{y}+|\xi|\right) U^{-} f & =f \\
\gamma^{-} U^{-} f & =0
\end{aligned}
$$


and

$$
\begin{aligned}
\left(\partial_{y}-|\xi|\right) U^{+} f & =f, \\
\gamma^{+} U^{+} f & =0 .
\end{aligned}
$$

The following proposition holds.

Proposition 4.1. Let $f$ be of the form $f=\left|\xi^{\prime}\right| \tilde{f}$ with $\tilde{f} \in H_{T}^{l+1}$. Then $U^{+} f \in$ $H_{T}^{l}, U^{-} f \in H_{T}^{l}$, and the following estimates hold:

$$
\begin{aligned}
\left\|U^{+} f\right\|_{l, T} & \leq c\|\tilde{f}\|_{l+1, T}, \\
\left\|U^{-} f\right\|_{l, T} & \leq c\|\tilde{f}\|_{l+1, T} .
\end{aligned}
$$

The proof of the above proposition is a straightforward modification of the proof of a similar statement given in [1].

The following lemmas are crucial for our analysis.

Lemma 4.2. Suppose $g_{\tau}^{+} \in H_{T}^{\prime l}$ with $\left.g_{\tau}^{+}\right|_{t=0}=0$. Then $\gamma^{-} U^{+} F_{1}\left(g_{\tau}^{+}, 0\right) \in H_{T}^{\prime l}$. Moreover, there exists $\alpha<1$ and $T_{\alpha}>0$, independent of $g_{\tau}^{+}$, such that

$$
\left\|\gamma^{-} U^{+} F_{1}\left(g_{\tau}^{+}, 0\right)\right\|_{l, T_{\alpha}} \leq \alpha\left\|g_{\tau}^{+}\right\|_{l, T} .
$$

Lemma 4.3. Suppose $g_{\tau}^{-} \in H_{T}^{\prime l}$ with $\left.g_{\tau}^{-}\right|_{t=0}=0$. Then $\gamma^{-} U^{-} F_{1}\left(0, g_{\tau}^{-}\right) \in H_{T}^{\prime l}$. Moreover, there exists $\alpha<1$ and $T_{\alpha}>0$, independent of $g_{\tau}^{-}$, such that

$$
\left\|\gamma^{+} U^{-} F_{1}\left(0, g_{\tau}^{-}\right)\right\|_{l, T_{\alpha}} \leq \alpha\left\|g_{\tau}^{-}\right\|_{l, T} .
$$

The meaning of the estimate on the operator $U^{+} F_{1}$ given in Lemma 4.2 is the following. Give to $U^{+} F_{1}$ a boundary datum which is different from zero only at $y=+1$, and evaluate the norm of the trace at $y=-1$. Then, up to the (sufficiently small) time $T_{\alpha}$, one has that this norm is strictly less than the norm of the boundary datum at $y=+1$. Lemma 4.3 admits a similar interpretation. It is natural to expect that the time $T_{\alpha}$ up to which the estimates are valid grows to infinity when the diffusivity goes to zero. This will show up clearly during the proof of the above lemmas, which are given in the appendix.

4.2. The inverse elliptic operators. We introduce the following operator $\mathcal{L}=$ $\left(\mathcal{L}_{\tau}, \mathcal{L}_{N}\right):$

$$
\begin{aligned}
\mathcal{L}_{N}\left(g_{N}^{+}, g_{N}^{-}\right) & =\frac{\sinh [|\xi|(y+1)]}{\sinh [2|\xi|]} g_{N}^{+}+\frac{\sinh [|\xi|(1-y)]}{\sinh [2|\xi|]} g_{N}^{-}, \\
\mathcal{L}_{\tau}\left(g_{N}^{+}, g_{N}^{-}\right) & =\frac{\cosh [|\xi|(y+1)]}{\sinh [2|\xi|]} N^{\prime} g_{N}^{+}-\frac{\cosh [|\xi|(1-y)]}{\sinh [2|\xi|]} N^{\prime} g_{N}^{-},
\end{aligned}
$$

where $N^{\prime}=i \xi /|\xi|$. This operator has the property of being divergence-free and harmonic; moreover, one can easily see that it is the gradient of a scalar function. Therefore, it solves Oseen equations. Finally, it has the property that its normal component has $g_{N}^{+}$and $g_{N}^{-}$as boundary conditions at $y=+1$ and $y=-1$, respectively.

We now give some estimates on this operator. In these estimates we shall always suppose that the boundary data $g_{N}^{ \pm}$are such that $|\xi|^{-1} g_{N} \in H_{T}^{\prime l+1}$. This hypothesis, which we shall refer to as integrability of the normal influx (in fact it is related to assuming that the total influx from the boundary is bounded), is necessary to handle the fact that the operator $\mathcal{L}$ is singular when $\xi \rightarrow 0$. 
The proposition below gives an estimate of $\mathcal{L}$ in the spaces $L_{T}^{l}$ and $H_{T}^{l}$. The estimate in the space $L_{T}^{l}$ will be used in the next subsections in the construction of the Oseen operator. The estimate in the space $H_{T}^{l}$ will be used in section 6 in the analysis of the Euler equations.

Proposition 4.4. Let $g_{N}^{ \pm}$be such that $|\xi|^{-1} g_{N}^{ \pm} \in H_{T}^{\prime l+1}$. Then $\mathcal{L}\left(g_{N}^{+}, g_{N}^{-}\right) \in$ $L_{T}^{l} \cap H_{T}^{l}$. The following estimates hold:

$$
\begin{aligned}
\left\|\mathcal{L}\left(g_{N}^{+}, g_{N}^{-}\right)\right\|_{l, T} & \leq c\left[\left\||\xi|^{-1} g_{N}^{+}\right\|_{l+1, T}+\left\||\xi|^{-1} g_{N}^{-}\right\|_{l+1, T}\right] \\
\left|\mathcal{L}\left(g_{N}^{+}, g_{N}^{-}\right)\right|_{l, T} & \leq c\left[\left\||\xi|^{-1} g_{N}^{+}\right\|_{l+1, T}+\left\||\xi|^{-1} g_{N}^{-}\right\|_{l+1, T}\right] .
\end{aligned}
$$

The following lemmas will be useful in the estimates of the Oseen operator.

Lemma 4.5. Let $g_{N}^{+}$be such that $|\xi|^{-1} g_{N}^{+} \in H_{T}^{\prime l+1}$. Then $\gamma^{-} \mathcal{L}_{\tau}\left(g_{N}^{+}, 0\right) \in L_{T}^{l}$, and the following estimate holds:

$$
\left\|\gamma^{-} \mathcal{L}_{\tau}\left(g_{N}^{+}, 0\right)\right\|_{l, T} \leq \frac{1}{2}\left\||\xi|^{-1} g_{N}^{+}\right\|_{l+1, T}
$$

Lemma 4.6. Let $g_{N}^{-}$be such that $|\xi|^{-1} g_{N}^{-} \in H_{T}^{\prime l+1}$. Then $\gamma^{+} \mathcal{L}_{\tau}\left(0, g_{N}^{-}\right) \in L_{T}^{l}$, and the following estimate holds:

$$
\left\|\gamma^{+} \mathcal{L}_{\tau}\left(0, g_{N}^{-}\right)\right\|_{l, T} \leq \frac{1}{2}\left\||\xi|^{-1} g_{N}^{-}\right\|_{l+1, T}
$$

The meaning of Lemma 4.5 is the following. Give to the operator $\mathcal{L}_{\tau}$ a boundary datum which is nonzero only at $y=+1$. Then its trace at $y=-1$ is strictly less (half) than the datum at $y=+1$. A similar interpretation can be given to Lemma 4.6. The proof of the above two lemmas is a straightforward consequence of the fact that $\left|\frac{\left|\xi^{\prime}\right|}{\sinh 2\left|\xi^{\prime}\right|}\right| \leq \frac{1}{2}$.

4.3. The projection-convection-diffusion operator. Through the operators we have introduced in the previous sections, we can define the operator $\mathcal{M}^{-}$. The tangential and normal component are defined as follows:

$$
\begin{aligned}
& \mathcal{M}_{\tau}^{-}\left(g_{\tau}^{-}, g_{N}^{-}\right)=U^{-}|\xi| F_{1}\left(0, g_{\tau}^{-}-\gamma^{-} \mathcal{L}_{\tau}\left(0, g_{N}^{-}\right)\right)+F_{1}\left(0, g_{\tau}^{-}-\gamma^{-} \mathcal{L}_{\tau}\left(0, g_{N}^{-}\right)\right) \\
& \mathcal{M}_{N}^{-}\left(g_{\tau}^{-}, g_{N}^{-}\right)=U^{-}|\xi| N^{\prime} F_{1}\left(0, g_{\tau}^{-}-\gamma^{-} \mathcal{L}_{\tau}\left(0, g_{N}^{-}\right)\right)
\end{aligned}
$$

One can verify that the operator $\mathcal{M}^{-}$is divergence-free, satisfies the Oseen equations, and has zero normal boundary condition at $y=-1$ :

$$
\begin{aligned}
\left(\partial_{t}-\nu \Delta+U \partial_{x}\right) \mathcal{M}^{-}\left(g_{\tau}^{-}, g_{N}^{-}\right)+\nabla p & =0, \\
\nabla \cdot \mathcal{M}^{-}\left(g_{\tau}^{-}, g_{N}^{-}\right) & =0, \\
\gamma^{-} \mathcal{M}^{-}\left(g_{\tau}^{-}, g_{N}^{-}\right) & =\left(g_{\tau}^{-}-\gamma^{-} \mathcal{L}_{\tau}\left(0, g_{N}^{-}\right), 0\right), \\
\left.\mathcal{M}^{-}\left(g_{\tau}^{-}, g_{N}^{-}\right)\right|_{t=0} & =0 .
\end{aligned}
$$

One can analogously define the operator $\mathcal{M}^{+}$:

$$
\begin{aligned}
& \mathcal{M}_{\tau}^{+}\left(g_{\tau}^{+}, g_{N}^{+}\right)=U^{+}|\xi| F_{1}\left(g_{\tau}^{+}-\gamma^{+} \mathcal{L}_{\tau}\left(g_{N}^{+}, 0\right), 0\right)+F_{1}\left(g_{\tau}^{+}-\gamma^{+} \mathcal{L}_{\tau}\left(g_{N}^{+}, 0\right), 0\right) \\
& \mathcal{M}_{N}^{+}\left(g_{\tau}^{+}, g_{N}^{+}\right)=U^{+}|\xi| N^{\prime} F_{1}\left(g_{\tau}^{+}-\gamma^{+} \mathcal{L}_{\tau}\left(g_{N}^{+}, 0\right), 0\right)
\end{aligned}
$$


It has the property of being divergence-free and of solving Oseen equations with zero normal boundary condition at $y=1$, namely,

$$
\begin{aligned}
\left(\partial_{t}-\nu \Delta+U \partial_{x}\right) \mathcal{M}^{+}\left(g_{\tau}^{+}, g_{N}^{+}\right)+\nabla p & =0 \\
\nabla \cdot \mathcal{M}^{+}\left(g_{\tau}^{+}, g_{N}^{+}\right) & =0 \\
\gamma^{+} \mathcal{M}^{+}\left(g_{\tau}^{+}, g_{N}^{+}\right) & =\left(g_{\tau}^{+}-\gamma^{+} \mathcal{L}_{\tau}\left(g_{N}^{+}, 0\right), 0\right), \\
\left.\mathcal{M}^{-}\left(g_{\tau}^{+}, g_{N}^{+}\right)\right|_{t=0} & =0 .
\end{aligned}
$$

4.4. The half space Oseen operators. One can finally define the half space Oseen operators $\mathcal{O}^{+}$and $\mathcal{O}^{-}$. These operators solve the Oseen equations, are divergence-free, and have the right boundary condition at $y=+1$ and $y=-1$, respectively. Moreover, the trace of these operators, evaluated at $y=-1$ and $y=+1$, respectively, has norm strictly less than the boundary datum. This property, expressed in Propositions 4.5 and 4.6 below, makes them suitable for the iterative procedure of the next section.

The operator $\mathcal{O}^{+}$is defined as

$$
\begin{aligned}
& \mathcal{O}_{N}^{+}\left(g_{\tau}^{+}, g_{N}^{+}\right)=\mathcal{L}_{N}\left(g_{N}^{+}, 0\right)+\mathcal{M}_{N}^{+}\left(g_{\tau}^{+}, g_{N}^{+}\right) \\
& \mathcal{O}_{\tau}^{+}\left(g_{\tau}^{+}, g_{N}^{+}\right)=\mathcal{L}_{\tau}\left(g_{N}^{+}, 0\right)+\mathcal{M}_{\tau}^{+}\left(g_{\tau}^{+}, g_{N}^{+}\right)
\end{aligned}
$$

The operator $\mathcal{O}^{-}$is defined as

$$
\begin{aligned}
& \mathcal{O}_{\tau}^{-}\left(g_{\tau}^{-}, g_{N}^{-}\right)=\mathcal{L}_{\tau}\left(0, g_{N}^{-}\right)+\mathcal{M}_{\tau}^{-}\left(g_{\tau}^{-}, g_{N}^{-}\right), \\
& \mathcal{O}_{N}^{-}\left(g_{\tau}^{-}, g_{N}^{-}\right)=\mathcal{L}_{N}\left(0, g_{N}^{-}\right)+\mathcal{M}_{N}^{-}\left(g_{\tau}^{-}, g_{N}^{-}\right) .
\end{aligned}
$$

These operators have the property of solving the Oseen equations in the channel: $\mathcal{O}^{+}$ with the right boundary condition at $y=+1 ; \mathcal{O}^{-}$with the right boundary condition at $y=-1$.

$$
\begin{aligned}
\left(\partial_{t}-\nu \Delta+U \partial_{x}\right) \mathcal{O}^{ \pm}\left(g_{\tau}^{ \pm}, g_{N}^{ \pm}\right)+\nabla p & =0 \\
\nabla \cdot \mathcal{O}^{ \pm}\left(g_{\tau}^{ \pm}, g_{N}^{ \pm}\right) & =0 \\
\gamma^{ \pm} \mathcal{O}^{ \pm}\left(g_{\tau}^{ \pm}, g_{N}^{ \pm}\right) & =\left(g_{\tau}^{ \pm}, g_{N}^{ \pm}\right), \\
\left.\mathcal{O}^{ \pm}\left(g_{\tau}^{-}, g_{N}^{-}\right)\right|_{t=0} & =0 .
\end{aligned}
$$

We now give some estimates on these operators. In the next section these estimates will allow us to construct the solution of the Oseen equation in the channel. In these estimates we shall always suppose that the normal component of the datum is such that $|\xi|^{-1} g_{N} \in H_{T}^{\prime l+1}$, i.e., that $\boldsymbol{g} \in \boldsymbol{H}_{T}^{\prime l}$.

Proposition 4.7. Let $\boldsymbol{g}^{+} \in \boldsymbol{H}_{T}^{\prime l}$ with $\left.\boldsymbol{g}^{+}\right|_{t=0}=0$. Then $\mathcal{O}^{+}\left(\boldsymbol{g}^{+}\right) \in L_{T}^{l}$ and the following estimate holds:

$$
\left\|\mathcal{O}^{+}\left(\boldsymbol{g}^{+}\right)\right\|_{l, T} \leq c\left|\boldsymbol{g}^{+}\right|_{l, T} .
$$

Proposition 4.8. Let $\boldsymbol{g}^{-} \in \boldsymbol{H}_{T}^{\prime l}$ with $\left.\boldsymbol{g}^{-}\right|_{t=0}=0$. Then $\mathcal{O}^{-}\left(\boldsymbol{g}^{-}\right) \in \boldsymbol{H}_{T}^{l}$ and the following estimate holds:

$$
\left\|\mathcal{O}^{-}\left(\boldsymbol{g}^{-}\right)\right\|_{l, T} \leq c\left|\boldsymbol{g}^{-}\right|_{l, T}
$$

The proof of these propositions can be easily achieved by using Propositions 4.4, 4.1, and 3.3. 
We now give the estimates on the trace of the operators $\mathcal{O}^{+}$and $\mathcal{O}^{-}$. We shall also prove that the trace at $y=-1$ (at $y=+1$, respectively) of $\mathcal{O}^{+}$(of $\mathcal{O}^{-}$, respectively) has the integrability property for the normal influx.

Proposition 4.9. Let $\boldsymbol{g}^{+} \in \boldsymbol{H}_{T}^{\prime l}$ with $\left.\boldsymbol{g}^{+}\right|_{t=0}=0$. Then $\gamma^{-} \mathcal{O}^{+} \in \boldsymbol{H}_{T}^{\prime l}$. Moreover, for any $0<\alpha<1$ there exists $T_{\alpha}>0$ such that

$$
\left|\gamma^{-} \mathcal{O}^{+}\left(\boldsymbol{g}^{+}\right)\right|_{l, T_{\alpha}} \leq \alpha\left|\boldsymbol{g}^{+}\right|_{l, T}
$$

Proposition 4.10. Let $\boldsymbol{g}^{-} \in \boldsymbol{H}_{T}^{\prime l}$ with $\left.\boldsymbol{g}^{-}\right|_{t=0}=0$. Then $\gamma^{+} \mathcal{O}^{-}\left(\boldsymbol{g}^{-}\right) \in \boldsymbol{H}_{T}^{\prime l}$. Moreover, for any $0<\alpha<1$ there exists $T_{\alpha}>0$ such that

$$
\left|\gamma^{+} \mathcal{O}^{-}\left(\boldsymbol{g}^{-}\right)\right|_{l, T_{\alpha}} \leq \alpha\left|\boldsymbol{g}^{-}\right|_{l, T}
$$

The proof of Propositions 4.9 and 4.10 is given in the appendix.

4.5. The Oseen operator with boundary data. We now have to solve the Oseen equations (4.1)-(4.5). We shall construct the solution as an infinite sum:

$$
\boldsymbol{u}=\sum_{i=0}^{\infty} \boldsymbol{u}^{(i)}
$$

Each term of the series solves the Oseen equations. The zeroth term $\boldsymbol{u}^{(0)}$ has the right boundary condition at $y=-1$ :

$$
\boldsymbol{u}^{(0)}=\mathcal{O}^{-}\left(\boldsymbol{g}^{-}\right)
$$

As the boundary condition for the first term of the series we choose $\gamma^{+} \boldsymbol{u}^{(1)}=\boldsymbol{g}^{+}-$ $\gamma^{+} \boldsymbol{u}^{(0)}$. Therefore, one has the following expression for $\boldsymbol{u}^{(1)}$ :

$$
\boldsymbol{u}^{(1)}=\mathcal{O}^{+}\left(\boldsymbol{g}^{+}-\gamma^{+} \boldsymbol{u}^{(0)}\right)
$$

In fact this choice will fix the boundary condition at $y=+1$; on the other end it generates a boundary datum at $y=-1$. We define the second term of the series $\boldsymbol{u}^{(2)}$ so that it cancels this boundary datum at $y=-1$ :

$$
\boldsymbol{u}^{(2)}=\mathcal{O}^{-}\left(-\gamma^{-} \boldsymbol{u}^{(1)}\right) .
$$

Recursively we define the generic even and odd term of the series:

$$
\begin{aligned}
\boldsymbol{u}^{(2 m)} & =\mathcal{O}^{-}\left(-\gamma^{-} \boldsymbol{u}^{(2 m-1)}\right), & & m>1, \\
\boldsymbol{u}^{(2 m+1)} & =\mathcal{O}^{+}\left(-\gamma^{+} \boldsymbol{u}^{(2 m)}\right), & & m>1 .
\end{aligned}
$$

To prove the convergence of the series we prove the following proposition.

Proposition 4.11. Let $\boldsymbol{g}^{ \pm} \in \boldsymbol{H}_{T}^{\prime l}$ with $\left.\boldsymbol{g}^{ \pm}\right|_{t=0}=0$. Then for any $0<\alpha<1$ there exists $T_{\alpha}>0$ such that for each $\boldsymbol{u}^{(i)}$ the following estimate holds:

$$
\left\|\boldsymbol{u}^{(i)}\right\|_{l, T_{\alpha}} \leq c \alpha^{i-2}\left(\left|\boldsymbol{g}^{+}\right|_{l, T}+\left|\boldsymbol{g}^{-}\right|_{l, T}\right)
$$


Proof. We prove the proposition when $i=2 \mathrm{~m}$. The proof is analogous when $i$ is odd:

$$
\begin{aligned}
\boldsymbol{u}^{(2 m)} & =\mathcal{O}^{-}\left(-\gamma^{-} \boldsymbol{u}^{(2 m-1)}\right) \\
& =\mathcal{O}^{-}\left(-\gamma^{-} \mathcal{O}^{+}\left(-\gamma^{+} \boldsymbol{u}^{(2 m-2)}\right)\right) \\
& =\cdots \\
& =\mathcal{O}^{-} \underbrace{\left(-\gamma^{-} \mathcal{O}^{+}\left(-\gamma^{+} \mathcal{O}^{-}\left(-\gamma^{-} \mathcal{O}^{+}\left(\cdots \left(-\gamma^{-} \mathcal{O}^{+}\right.\right.\right.\right.\right.}_{2 m-2 \text { times }}\left(\boldsymbol{g}^{+}-\gamma^{+} \mathcal{O}^{-} \boldsymbol{g}^{-}\right)) \ldots))) .
\end{aligned}
$$

Hence, first using Proposition 4.8, then using Propositions 4.9 and 4.10, one has

$$
\begin{aligned}
\left\|\boldsymbol{u}^{(2 m)}\right\|_{l, T_{\alpha}} & =\left\|\mathcal{O}^{-}\left(-\gamma^{-} \boldsymbol{u}^{(2 m-1)}\right)\right\|_{l, T_{\alpha}} \\
& \leq c\left|\gamma^{-} \boldsymbol{u}^{(2 m-1)}\right|_{l, T_{\alpha}}=c\left|\gamma^{-} \mathcal{O}^{+}\left(\gamma^{+} \boldsymbol{u}^{(2 m-2)}\right)\right|_{l, T_{\alpha}} \\
& \leq c \alpha\left|\gamma^{+} \boldsymbol{u}^{(2 m-2)}\right|_{l, T_{\alpha}} \\
& \leq \cdots \\
& \leq c \alpha^{2 m-2}\left(\left|\boldsymbol{g}^{+}\right|_{l, T}+\left|\boldsymbol{g}^{-}\right|_{l, T}\right)
\end{aligned}
$$

Therefore the series (4.16) with $\boldsymbol{u}^{(i)}$ given by (4.17)-(4.21) is convergent, and we can define the operator $\mathcal{O}_{b}$, solving Oseen equations with boundary data:

$$
\mathcal{O}_{b}\left(\boldsymbol{g}^{-}, \boldsymbol{g}^{+}\right)=\sum_{i=0}^{\infty} \boldsymbol{u}^{(i)}
$$

The following theorem is the main result of this section.

THEOREM 4.12. Let $\boldsymbol{g}^{ \pm}=\left(g_{\tau}^{ \pm}, g_{N}^{ \pm}\right)$such that $\boldsymbol{g}_{N}^{ \pm} \in \boldsymbol{H}_{T}^{\prime l}$ and $\left.\boldsymbol{g}^{ \pm}\right|_{t=0}=0$. Then there exists $T_{\alpha}$, independent of $\boldsymbol{g}^{ \pm}$, such that the operator $\mathcal{O}_{b}$, defined by (4.23) with $\boldsymbol{u}^{(i)}$ given by (4.17)-(4.21), represents the solutions of the Oseen equations (4.1)-(4.5). Moreover, $\mathcal{O}_{b}\left(\boldsymbol{g}^{-}, \boldsymbol{g}^{+}\right) \in L_{T_{\alpha}}^{l}$ and the following estimate holds:

$$
\left\|\mathcal{O}_{b}\left(\boldsymbol{g}^{-}, \boldsymbol{g}^{+}\right)\right\|_{l, T_{\alpha}} \leq c\left[\left|\boldsymbol{g}^{+}\right|_{l, T}+\left|\boldsymbol{g}^{-}\right|_{l, T}\right] .
$$

5. The explicit solution of the Oseen equations. We now solve the Oseen equations with source term, boundary data, and initial data (1.1)-(1.5). To accomplish this task first we introduce the projection operator onto the divergence-free part of a vector function. We shall write this projection operator so that its normal component evaluated at the boundary is identically zero. Then we shall use this projection operator to project the convection-diffusion operator $F_{0}$ and $F_{2}$ to get the operators $\mathcal{O}_{i}$ and $\mathcal{O}_{s}$. These operators solve the Oseen equation with initial datum and source term, respectively. On the other hand, they generate wrong boundary data. Finally, in the last subsection we shall use the operator $\mathcal{O}_{b}$ to cancel the wrong boundary data and get the representation of the solution of (1.1)-(1.5).

5.1. The projection operator. We introduce the following projection operator:

$$
P=1-\nabla \Delta_{N}^{-1} \nabla
$$

In the above expression with $\Delta_{N}^{-1}$ we have denoted the operator that solves the Poisson equation with Neumann boundary conditions. We give the explicit expression of this 
projection operator:

$$
\begin{aligned}
P_{\tau} \boldsymbol{w}= & w_{\tau}+\frac{N^{\prime}}{\sinh \left(2\left|\xi^{\prime}\right|\right)}\left[\gamma^{+} w_{N} \cosh \left[\left|\xi^{\prime}\right|(y+1)\right]-\gamma^{-} w_{N} \cosh \left[\left|\xi^{\prime}\right|(y-1)\right]\right] \\
& -\frac{\left|\xi^{\prime}\right|}{2 \sinh \left(2\left|\xi^{\prime}\right|\right)}\left\{\int_{-1}^{y} d y^{\prime}\left[\cosh \left(\left|\xi^{\prime}\right|\left(y-y^{\prime}-2\right)\right)+\cosh \left(\left|\xi^{\prime}\right|\left(y+y^{\prime}\right)\right)\right] w_{\tau}\left(\left|\xi^{\prime}\right|, y^{\prime}\right)\right. \\
& -\int_{-1}^{y} d y^{\prime}\left[\sinh \left(\left|\xi^{\prime}\right|\left(y-y^{\prime}-2\right)\right)-\sinh \left(\left|\xi^{\prime}\right|\left(y+y^{\prime}\right)\right)\right] N^{\prime} w_{N}\left(\left|\xi^{\prime}\right|, y^{\prime}\right) \\
& +\int_{y}^{1} d y^{\prime}\left[\cosh \left(\left|\xi^{\prime}\right|\left(y-y^{\prime}+2\right)\right)+\cosh \left(\left|\xi^{\prime}\right|\left(y+y^{\prime}\right)\right)\right] w_{\tau}\left(\left|\xi^{\prime}\right|, y^{\prime}\right) \\
& \left.-\int_{y}^{1} d y^{\prime}\left[\sinh \left(\left|\xi^{\prime}\right|\left(y-y^{\prime}+2\right)\right)-\sinh \left(\left|\xi^{\prime}\right|\left(y+y^{\prime}\right)\right)\right] N^{\prime} w_{N}\left(\left|\xi^{\prime}\right|, y^{\prime}\right)\right\}, \\
P_{N} \boldsymbol{w}= & \frac{1}{\sinh \left(2\left|\xi^{\prime}\right|\right)}\left\{\gamma^{+} w_{N} \sinh \left[\left|\xi^{\prime}\right|(y+1)\right]-\gamma^{-} w_{N} \sinh \left[\left|\xi^{\prime}\right|(y-1)\right]\right\} \\
& +\frac{\left|\xi^{\prime}\right|}{2 \sinh \left(2\left|\xi^{\prime}\right|\right)}\left\{\int_{-1}^{y} d y^{\prime}\left[\sinh \left(\left|\xi^{\prime}\right|\left(y-y^{\prime}-2\right)\right)+\sinh \left(\left|\xi^{\prime}\right|\left(y+y^{\prime}\right)\right)\right] N^{\prime} w_{\tau}\right. \\
& +\int_{-1}^{y} d y^{\prime}\left[\cosh \left(\left|\xi^{\prime}\right|\left(y-y^{\prime}-2\right)\right)-\cosh \left(\left|\xi^{\prime}\right|\left(y+y^{\prime}\right)\right)\right] w_{N} \\
& +\int_{y}^{1} d y^{\prime}\left[\sinh \left(\left|\xi^{\prime}\right|\left(y-y^{\prime}+2\right)\right)+\sinh \left(\left|\xi^{\prime}\right|\left(y+y^{\prime}\right)\right)\right] N^{\prime} w_{\tau} \\
& \left.+\int_{y}^{1} d y^{\prime}\left[\cosh \left(\left|\xi^{\prime}\right|\left(y-y^{\prime}+2\right)\right)-\cosh \left(\left|\xi^{\prime}\right|\left(y+y^{\prime}\right)\right)\right] w_{N}\right\} .
\end{aligned}
$$

It is not difficult to see that the following proposition holds.

Proposition 5.1. Let $\boldsymbol{w} \in L_{T}^{l}$. Then $P \boldsymbol{w} \in L_{T}^{l}$ and

$$
\|P \boldsymbol{w}\|_{l, T} \leq c\|\boldsymbol{w}\|_{l, T}
$$

It is important to notice that, if $\gamma^{ \pm} w_{N}=0$, then the normal component of the projection operator evaluated at the boundary is identically zero:

$$
\text { if } \gamma^{ \pm} w_{N}=0, \quad \text { then } \gamma^{ \pm} P_{N} \boldsymbol{w}=0 .
$$

5.2. The projected convection-diffusion operators. We can now introduce the operators $\mathcal{O}_{i}$ and $\mathcal{O}_{s}$, defined as

$$
\mathcal{O}_{i}=P F_{0}, \quad \mathcal{O}_{s}=P F_{2} .
$$

Supposing that $\boldsymbol{\nabla} \cdot \boldsymbol{u}_{0}=0$, one therefore has that $\mathcal{O}_{i} \boldsymbol{u}_{0}$ satisfies

$$
\begin{aligned}
\left(\partial_{t}-\partial_{Y Y}-\varepsilon^{2} \partial_{x x}\right) \mathcal{O}_{i} \boldsymbol{u}_{0}+\nabla p & =0, \\
\boldsymbol{\nabla} \cdot \mathcal{O}_{i} \boldsymbol{u}_{0} & =0, \\
\left.\mathcal{O}_{i} \boldsymbol{u}_{0}\right|_{t=0} & =\boldsymbol{u}_{0} .
\end{aligned}
$$

On the other hand, $\mathcal{O}_{s} \boldsymbol{w}$ satisfies

$$
\begin{aligned}
\left(\partial_{t}-\partial_{Y Y}-\varepsilon^{2} \partial_{x x}\right) \mathcal{O}_{s} \boldsymbol{w}+\nabla p & =\boldsymbol{w} \\
\boldsymbol{\nabla} \cdot \mathcal{O}_{s} \boldsymbol{w} & =0 \\
\left.\mathcal{O}_{s} \boldsymbol{w}\right|_{t=0} & =0 .
\end{aligned}
$$


The following estimates are a consequence of the properties of $P$, expressed in Proposition 5.1, and of the properties of $F_{0}$ and $F_{2}$, expressed in Propositions 3.1 and 3.4.

Proposition 5.2. Let $\boldsymbol{u}_{0} \in L^{l}$. Then $\mathcal{O}_{i} \boldsymbol{u}_{0} \in L_{T}^{l}$ and

$$
\left\|\mathcal{O}_{i} \boldsymbol{u}_{0}\right\|_{l, T} \leq\left\|\boldsymbol{u}_{0}\right\|_{l} \text {. }
$$

Proposition 5.3. Let $\boldsymbol{w} \in L_{T}^{l}$. Then $\mathcal{O}_{s} \boldsymbol{w} \in L_{T}^{l}$ and

$$
\left\|\mathcal{O}_{s} \boldsymbol{w}\right\|_{l, T} \leq c\|\boldsymbol{w}\|_{l, T} .
$$

The normal components of the operators $\mathcal{O}_{i}$ and $\mathcal{O}_{s}$ evaluated at the boundary are zero (because of (5.2)). This readily gives the following estimates on the traces of $\mathcal{O}_{i}$ and $\mathcal{O}_{s}$.

Proposition 5.4. Let $\boldsymbol{u}_{0} \in L^{l}$. Then $\gamma^{ \pm} \mathcal{O}_{i} \boldsymbol{u}_{0} \in \boldsymbol{H}_{T}^{\prime l}$ and

$$
\left|\gamma^{ \pm} \mathcal{O}_{i} \boldsymbol{u}_{0}\right|_{l, T} \leq c\left\|\boldsymbol{u}_{0}\right\|_{l} .
$$

Proposition 5.5. Let $\boldsymbol{w} \in L_{T}^{l}$. Then $\gamma^{ \pm} \mathcal{O}_{s} \boldsymbol{w} \in \boldsymbol{H}_{T}^{\prime l}$ and

$$
\left|\gamma^{ \pm} \mathcal{O}_{s} \boldsymbol{w}\right|_{l, T} \leq c\|\boldsymbol{w}\|_{l, T}
$$

5.3. The solution of the Oseen equations. We can finally introduce the operator $\mathcal{O}$ that solves $(1.1)-(1.5)$ :

$$
\mathcal{O}\left(\boldsymbol{f}, \boldsymbol{u}_{0}, \boldsymbol{g}^{-}, \boldsymbol{g}^{+}\right)
$$

$$
=\mathcal{O}_{s} \boldsymbol{f}+\mathcal{O}_{i} \boldsymbol{u}_{0}+\mathcal{O}_{b}\left(\boldsymbol{g}^{+}-\gamma^{+} \mathcal{O}_{s} \boldsymbol{f}-\gamma^{+} \mathcal{O}_{i} \boldsymbol{u}_{0}, \boldsymbol{g}^{-}-\gamma^{-} \mathcal{O}_{s} \boldsymbol{f}-\gamma^{-} \mathcal{O}_{i} \boldsymbol{u}_{0}\right) .
$$

Therefore, if one defines

$$
\boldsymbol{u}=\mathcal{O}\left(\boldsymbol{f}, \boldsymbol{u}_{0}, \boldsymbol{g}^{+}, \boldsymbol{g}^{-}\right),
$$

then one has that $\boldsymbol{u}$ solves the system (1.1)-(1.5).

Remark 5.1. The representation of the solution given by the operator $\mathcal{O}$ is valid only up to the time $T_{\alpha}$. In fact the series which defines the operator $\mathcal{O}_{b}$ converges only up to the time $T_{\alpha}$. On the other hand, given that $T_{\alpha}$ does not depend on the boundary data, one can take the value of the solution at the time $T_{\alpha}$ as the initial datum and solve the corresponding Oseen problem up to the time $2 T_{\alpha}$. One can therefore construct the solution up to the time $T$. Moreover, we also notice that the time $T_{\alpha}=O\left(\nu^{-1}\right)$. Therefore, in the zero viscosity limit, the representation (5.4) is valid for an arbitrarily long time. The following theorem holds.

TheOREm 5.6. Suppose $\boldsymbol{f} \in L_{T}^{l}, \boldsymbol{u}_{0} \in L^{l}$, and $\boldsymbol{g}^{ \pm} \in \boldsymbol{H}_{T}^{\prime l}$. Suppose the following compatibility conditions are verified:

$$
\nabla \cdot u_{0}=0
$$

and

$$
\left.\boldsymbol{g}^{ \pm}\right|_{t=0}=\gamma^{ \pm} \boldsymbol{u}_{0}
$$

Then the solution of the Oseen equations (1.1)-(1.5) is represented, for a time $T_{\alpha}$, by (5.4). Moreover, the following estimate holds:

$$
\left\|\mathcal{O}\left(\boldsymbol{f}, \boldsymbol{u}_{0}, \boldsymbol{g}^{-}, \boldsymbol{g}^{+}\right)\right\|_{l, T} \leq c\left[\|\boldsymbol{f}\|_{l, T}+\left\|\boldsymbol{u}_{0}\right\|_{l}+\left|\boldsymbol{g}^{+}\right|_{l, T}+\left|\boldsymbol{g}^{-}\right|_{l, T}\right] .
$$


The proof of this theorem is a consequence of Propositions 5.2, 5.3, 5.4, and 5.5 and Theorem 4.12 .

We finally give a proposition which will be useful in the solution of the error equation, given in the next section. It says that if the forcing term in (1.1) is of the same order of the square root of the viscosity, then one can use the heat kernel to increase the regularity of the solution.

Proposition 5.7. Suppose $\boldsymbol{f}=\sqrt{\nu} \boldsymbol{h}$ with $\boldsymbol{h} \in L_{T}^{l-1}, \boldsymbol{g}^{ \pm} \in \boldsymbol{H}_{T}^{\prime l}$, and $\boldsymbol{u}_{0} \in$ $L^{\prime l}$. Suppose that the compatibility conditions (5.5) and (5.6) are satisfied. Then $\mathcal{O}_{s}\left(\boldsymbol{f}, \boldsymbol{g}^{-}, \boldsymbol{g}^{+}\right) \in L_{T}^{l}$, and the following estimate holds:

$$
\left\|\mathcal{O}\left(\boldsymbol{f}, \boldsymbol{u}_{0}, \boldsymbol{g}^{+}, \boldsymbol{g}^{-}\right)\right\|_{l, T} \leq c\left[\|\boldsymbol{h}\|_{l-1, T}+\left\|\boldsymbol{u}_{0}\right\|_{l}+\left|\boldsymbol{g}^{+}\right|_{l, T}+\left|\boldsymbol{g}^{-}\right|_{l, T}\right] .
$$

The proof of this proposition can be easily achieved using the analogous estimate for the operator $F_{2}$ given in Proposition 3.5, and the estimates for $P, \mathcal{O}_{s}, \mathcal{O}_{i}$, and $\mathcal{O}_{b}$, given in Propositions 5.1, 5.2, 5.3, 5.4, and 5.5 and Theorem 4.12.

6. The asymptotic analysis. We are now ready to introduce the boundary layer analysis for the Oseen equations (1.1)-(1.5). For simplicity we shall suppose that the source term is not present. In a remark at the end of section 6.1 we shall sketch the procedure that allows us to handle a source term.

We shall impose the initial condition $\boldsymbol{u}_{0} \in H^{l}$ and show that the solution is the sum of an inviscid (Euler) part, two boundary layers ( Prandtl) parts exponentially decaying outside a region of size $\varepsilon=\sqrt{\nu}$ close to the two boundaries $y=-1$ and $y=+1$, and a correction term.

We seek a solution of the form

$$
\begin{aligned}
& u=u^{E}+u^{P}+\varepsilon w_{\tau}, \\
& v=v^{E}+\varepsilon v^{P}+\varepsilon w_{N}, \\
& p=p^{E}+\varepsilon p^{w}
\end{aligned}
$$

where $\left(u^{E}, v^{E}\right)$ represents the inviscid solution, $\left(u^{P}, \varepsilon v^{P}\right)$ represents the Prandtl part which describes the behavior of the fluid in the boundary layers close to $y= \pm 1$, and $\left(w_{\tau}, w_{N}\right)$ is the correction term. They solve the following equations.

1. The convective equations

$$
\begin{aligned}
\left(\partial_{t}+U \partial_{x}\right) \boldsymbol{u}^{E}+\nabla p^{E} & =0 \\
\nabla \cdot \boldsymbol{u}^{E} & =0, \\
\gamma^{-} v^{E} & =g_{N}^{-}, \\
\gamma^{+} v^{E} & =g_{N}^{+}, \\
\boldsymbol{u}^{E}(t=0) & =\boldsymbol{u}_{0} .
\end{aligned}
$$

2. The boundary layer equations

$$
\begin{aligned}
\left(\partial_{t}-\varepsilon^{2} \Delta+U \partial_{x}\right) u^{P} & =0, \\
\partial_{x} u^{P}+\partial_{Y} v^{P} & =0, \\
\gamma^{-} u^{P} & =g_{\tau}^{-}-\gamma^{-} u^{E}, \\
\gamma^{+} u^{P} & =g_{\tau}^{+}-\gamma^{+} u^{E}, \\
u^{P}(t=0) & =0 .
\end{aligned}
$$


3. The correction equations

$$
\begin{aligned}
\left(\partial_{t}-\varepsilon^{2} \Delta+U \partial_{x}\right) \boldsymbol{w}+\boldsymbol{\nabla} p^{w} & =\varepsilon \Delta \boldsymbol{u}^{E}, \\
\boldsymbol{\nabla} \cdot \boldsymbol{w} & =0, \\
\gamma^{-} \boldsymbol{w} & =\left(0,-\gamma^{-} v^{P}\right), \\
\gamma^{+} \boldsymbol{w} & =\left(0,-\gamma^{+} v^{P}\right), \\
\boldsymbol{w}(t=0) & =0 .
\end{aligned}
$$

We require to hold the compatibility conditions between the boundary and the initial data:

$$
\begin{aligned}
\gamma^{ \pm} v_{0} & =g_{N}^{ \pm}(x, t=0), \\
g_{\tau}^{ \pm}(t=0) & =-\gamma^{ \pm} u_{0} .
\end{aligned}
$$

We require to hold the incompressibility condition for the initial data:

$$
\boldsymbol{\nabla} \cdot \boldsymbol{u}_{0}=0
$$

Moreover, the incompressibility condition requires that

$$
\int_{-\infty}^{\infty}\left(g_{N}^{+}-g_{N}^{-}\right) d x=0
$$

Notice the absence of the pressure term in (6.9)-(6.13). This is due to the introduction of a boundary layer corrector which differs from the usual Prandtl velocity for the value of the Euler velocity at the boundary. Namely, $\tilde{\boldsymbol{u}}^{P}=\boldsymbol{u}^{P}-\gamma \boldsymbol{u}^{E}$. Then, using the Euler equation at the boundary, one gets (6.9). For more details, see [2].

We now solve the above equations.

6.1. The Euler equations. One can see that the solution of (6.4)-(6.8) is

$$
\begin{aligned}
v^{E}= & \mathcal{L}_{N}\left(g_{N}^{+}-v_{0}^{E}(x-U t, 1), g_{N}^{-}-v_{0}^{E}(x-U t,-1)\right) \\
& +v_{0}(x-U t, y), \\
& \\
u^{E}= & \mathcal{L}_{\tau}\left(g_{N}^{+}-v_{0}^{E}(x-U t, 1), g_{N}^{-}-v_{0}^{E}(x-U t,-1)\right) \\
+ & u_{0}(x-U t, y),
\end{aligned}
$$

where the operator $\mathcal{L}$ was introduced in section 4.2 . We now give an estimate on the above solution.

Proposition 6.1. Let $\boldsymbol{g} \in \boldsymbol{H}_{T}^{\prime l}$. Moreover, let $\boldsymbol{u}_{0} \in H^{l}$ satisfy the compatibility conditions (6.19) and (6.20) and the incompressibility condition (6.21). Then $\boldsymbol{u}^{E} \in$ $H_{T}^{l}$ and the following estimate holds:

$$
\left|\boldsymbol{u}^{E}\right|_{l, T} \leq c\left(\left|\boldsymbol{g}^{-}\right|_{l, T}+\left|\boldsymbol{g}^{+}\right|_{l, T}+\left|\boldsymbol{u}_{0}\right|_{l}\right) .
$$

The proof is based on Proposition 4.4.

Remark 6.1. If a source term $f$ is present in (6.4) one can solve the inviscid equation using the integrated (with respect to time) projection operator onto the divergence-free part. 
6.2. The Prandtl equations. Equations (6.9) and the boundary conditions (6.11) and (6.12) and the initial condition (6.13) are convection-diffusion equations with boundary data and zero initial datum and no source. We have already solved these equations through the operator $F_{1}$ introduced in section 3 . The solution is, therefore,

$$
u^{P}=F_{1}\left(g_{\tau}^{+}-\gamma^{+} u^{E}, g_{\tau}^{-}-\gamma^{-} u^{E}\right) .
$$

The normal component can be found using the incompressibility condition (6.10):

$$
v^{P}=\int_{Y}^{0} d Y^{\prime} \partial_{x} u^{P}
$$

Therefore, we conclude with the following proposition.

Proposition 6.2. Let $\mathbf{g}^{ \pm} \in \boldsymbol{H}_{T}^{\prime l}$ satisfy the compatibility conditions (6.19)(6.20) and the incompressibility condition (6.21). Then $u^{P} \in K_{T}^{l, \mu}, v^{P} \in K_{T}^{l-1, \mu}$, and the following estimates hold:

$$
\begin{aligned}
\left|u^{P}\right|_{l, \mu, T} & \leq c\left(\left|\mathbf{g}^{-}\right|_{l, T}+\left|\mathbf{g}^{+}\right|_{l, T}+\left|\boldsymbol{u}_{0}\right|_{l}\right), \\
\left|v^{P}\right|_{l-1, \mu, T} & \leq c\left(\left|\mathbf{g}^{-}\right|_{l, T}+\left|\mathbf{g}^{+}\right|_{l, T}+\left|\boldsymbol{u}_{0}\right|_{l}\right) .
\end{aligned}
$$

6.3. The error equation. Let us now consider the correction term $\boldsymbol{w}$ satisfying (6.14)-(6.18).

One can see that (6.14)-(6.18) are of the same form as (1.1)-(1.5), namely, they are the Oseen equations with source term and boundary and initial data satisfying the hypotheses of Proposition 5.7. In fact the source term in (6.14) is $\varepsilon \Delta e^{i \xi U t} \boldsymbol{u}_{0}$, which is of the form $\varepsilon \boldsymbol{h}$ with $\boldsymbol{h} \in H_{T}^{l-2}$. Moreover, the boundary data $-\gamma^{ \pm} v^{P}$ are of the form

$$
-\gamma^{ \pm} v^{P}=\partial_{x} \beta^{ \pm}=-|\xi| N^{\prime} \beta^{ \pm}, \quad \text { where } \quad \beta^{ \pm}=\int_{0}^{ \pm 1 / \varepsilon} d Y^{\prime} u^{P( \pm)} \in H_{T}^{\prime l} .
$$

Therefore, $\gamma^{ \pm} \boldsymbol{w} \in \boldsymbol{H}_{T}^{\prime l-1}$.

Hence the solution has the form given by (5.4) and we can give the estimate on the error $\boldsymbol{w}$.

Proposition 6.3. Let us suppose that $\boldsymbol{g}^{ \pm} \in \boldsymbol{H}_{T}^{\prime l}$ and $\boldsymbol{u}_{0} \in H^{l}$, satisfying the compatibility conditions (6.19)-(6.20) and the incompressibility condition (6.21). Then the solution of (6.14)-(6.18) $\boldsymbol{w} \in L_{T}^{l-1}$, and the following estimate hold:

$$
\|w\|_{l-1, T} \leq c\left[\left|\boldsymbol{g}^{+}\right|_{l, T}+\left|\boldsymbol{g}^{-}\right|_{l, T}+\left|\boldsymbol{u}_{0}\right|_{l}\right] .
$$

The above estimate is not enough to get the convergence of the solution of the Oseen equations to $\boldsymbol{u}^{E}+\boldsymbol{u}^{P}$ in a space where first derivatives are considered. A more refined analysis of the structure of the error is needed.

6.4. The structure of the error. We divide the error in the following way:

$$
\boldsymbol{w}=\boldsymbol{w}^{E}+\boldsymbol{w}^{B L}+\varepsilon \boldsymbol{e} .
$$

The Eulerian part of the error $\boldsymbol{w}^{E}$ satisfies convective equations, of the type (6.4)-(6.8), with $\varepsilon \Delta u^{E}$ as source term and with prescribed normal boundary data $-\gamma^{ \pm} v^{P}$. 
The boundary layer part of the error $\boldsymbol{w}^{B L}$ satisfies the boundary layer equations, of the type (6.9)-(6.13), with prescribed tangential component $-\gamma^{ \pm} \boldsymbol{w}^{E}$.

Finally the overall correction $\boldsymbol{e}$ satisfies the correction equations, of the type (6.14)-(6.18), with zero source term and with prescribed boundary data $\left(0,-\gamma^{ \pm} w_{N}^{B L}\right)$, where with $w_{N}^{B L}$ we have denoted the normal component of $\boldsymbol{w}^{B L}$.

One can therefore state the following proposition.

Proposition 6.4. Suppose the hypotheses of Proposition 6.3 hold true. Then the correction $\boldsymbol{w}$ admits a decomposition of the form (6.26) with $\boldsymbol{w}^{E} \in H_{T}^{l-1}, w_{\tau}^{B L} \in$ $K_{T}^{l-1, \mu}, w_{N}^{B L} \in K_{T}^{l-2, \mu}, e \in L_{T}^{l-2}$, and the following estimate holds:

$\left|\boldsymbol{w}^{E}\right|_{l-1, T}+\left|w_{\tau}^{B L}\right|_{l-1, \mu, T}+\left|w_{N}^{B L}\right|_{l-2, \mu, T}+\|\boldsymbol{e}\|_{l-2, T} \leq c\left(\left|\boldsymbol{g}^{+}\right|_{l, T}+\left|\boldsymbol{g}^{-}\right|_{l, T}+\left|\boldsymbol{u}_{0}\right|_{l}\right)$.

6.5. The main results. We can summarize the results of this section in the following theorem which is the main result of this paper.

TheOREM 6.5. Let $\boldsymbol{g}^{ \pm} \in \boldsymbol{H}_{T}^{\prime l}$ and $\boldsymbol{u}_{0} \in H^{l}$ satisfy the compatibility conditions (6.19)-(6.20) and the incompressibility condition (6.21). Then the solution of Oseen equations (1.1)-(1.5) can be written in the form

$$
\boldsymbol{u}=\boldsymbol{u}^{E}+\boldsymbol{u}^{P}+\varepsilon \boldsymbol{w},
$$

where $\boldsymbol{u}^{E}$ satisfies (6.4)-(6.8), $\boldsymbol{u}^{P}$ satisfies (6.9)-(6.13), and $\boldsymbol{w}$ satisfies (6.14)-(6.18). Moreover, $\boldsymbol{u}^{E} \in H_{T}^{l}, u^{P} \in K_{T}^{l, \mu}, v^{P} \in K_{T}^{l-1, \mu}$, and $\boldsymbol{w} \in L_{T}^{l-1}$. Moreover, the correction can be decomposed as

$$
\boldsymbol{w}=\boldsymbol{w}^{E}+\boldsymbol{w}^{B L}+\varepsilon \boldsymbol{e}
$$

with $\boldsymbol{w}^{E} \in H_{T}^{l-1}, w_{\tau}^{B L} \in K_{T}^{l-1, \mu}, w_{N}^{B L} \in K_{T}^{l-2, \mu}$, and $\boldsymbol{e} \in L_{T}^{l-2}$. The following estimates hold:

$$
\begin{aligned}
\left|\boldsymbol{u}^{E}\right|_{l, T} & \leq c\left(\left|\boldsymbol{g}^{+}\right|_{l, T}+\left|\boldsymbol{g}^{-}\right|_{l, T}+\left|\boldsymbol{u}_{0}\right|_{l}\right), \\
\left|u^{P}\right|_{l, \mu, T} & \leq c\left(\left|\boldsymbol{g}^{+}\right|_{l, T}+\left|\boldsymbol{g}^{-}\right|_{l, T}+\left|\boldsymbol{u}_{0}\right|_{l}\right), \\
\left|v^{P}\right|_{l-1, \mu, T} & \leq c\left(\left|\boldsymbol{g}^{+}\right|_{l, T}+\left|\boldsymbol{g}^{-}\right|_{l, T}+\left|\boldsymbol{u}_{0}\right|_{l}\right), \\
\left|\boldsymbol{w}^{E}\right|_{l-1, T} & \leq c\left(\left|\boldsymbol{g}^{+}\right|_{l, T}+\left|\boldsymbol{g}^{-}\right|_{l, T}+\left|\boldsymbol{u}_{0}\right|_{l}\right), \\
\left|w_{\tau}^{B L}\right|_{l-1, \mu, T} & \leq c\left(\left|\boldsymbol{g}^{+}\right|_{l, T}+\left|\boldsymbol{g}^{-}\right|_{l, T}+\left|\boldsymbol{u}_{0}\right|_{l}\right), \\
\left|w_{N}^{B L}\right|_{l-2, \mu, T} & \leq c\left(\left|\boldsymbol{g}^{+}\right|_{l, T}+\left|\boldsymbol{g}^{-}\right|_{l, T}+\left|\boldsymbol{u}_{0}\right|_{l}\right), \\
\|\boldsymbol{e}\|_{l-2, T} & \leq c\left(\left|\boldsymbol{g}^{+}\right|_{l, T}+\left|\boldsymbol{g}^{-}\right|_{l, T}+\left|\boldsymbol{u}_{0}\right|_{l}\right) .
\end{aligned}
$$

From the above theorem the following estimate on the convergence of the Oseen equation to $\boldsymbol{u}^{E}+\boldsymbol{u}^{P}$ easily follows.

Corollary 6.1. Let $\boldsymbol{u}_{0} \in H^{4}$ and $\boldsymbol{g}^{ \pm} \in L^{\infty}\left([0, T], H^{4}\right)$ satisfy the compatibility conditions (6.19)-(6.20) and the incompressibility condition (6.21). Moreover, suppose that the inflows at the boundaries $g_{N}^{ \pm}$are such that $|\xi|^{-1} g_{N}^{ \pm} \in L^{\infty}\left([0, T], L^{2}\right)$. If $\boldsymbol{u}, \boldsymbol{u}^{E}$, and $\boldsymbol{u}^{P}$ denote the solutions of (1.1)-(1.5), (6.4)-(6.8), and (6.9)-(6.13), respectively, then one has the following estimate:

$\left\|\boldsymbol{u}-\left(\boldsymbol{u}^{E}+\boldsymbol{u}^{P}\right)\right\|_{L^{\infty}\left([0, T], H^{2}\right)} \leq c \varepsilon^{1 / 2}\left[\left\|\boldsymbol{u}_{0}\right\|_{H^{4}}+\left\|\boldsymbol{g}^{+}\right\|_{L^{\infty}\left([0, T], H^{4}\right)}+\left\|\boldsymbol{g}^{-}\right\|_{L^{\infty}\left([0, T], H^{4}\right)}\right]$. (6.30) 
The factor $\varepsilon^{1 / 2}$ comes from the fact that the correction $\boldsymbol{w}$ also has a boundary layer structure, i.e., its derivative with respect to $y$ is $O\left(\varepsilon^{-1}\right)$ in a region of size $O\left(\varepsilon^{1 / 2}\right)$.

Remark 6.2. With minor formal modifications in the definitions of the function spaces $H^{l}$ given in section 2, which allow $l$ to be equal to 1 , Corollary 6.1 could be stated for initial data belonging to $H^{3}$.

\section{Appendix.}

Proof of Lemma 4.2. Let us consider first the $L^{2}$ norm.

$$
\begin{aligned}
& \sup _{0 \leq t \leq T}\left\|\gamma^{-} U^{+} F_{1}\left(g_{\tau}^{+}, 0\right)\right\|_{L^{2}\left(\xi^{\prime}\right)}^{2} \\
& =\sup _{0 \leq t \leq T}\left\|\int_{-1}^{1} d y^{\prime} e^{-\left|\xi^{\prime}\right|\left(1+y^{\prime}\right)} \int_{0}^{t} d s e^{-\left[\nu \xi^{2}+i \xi^{\prime} U\right](t-s)} \sum_{n=-\infty}^{\infty} \frac{y^{\prime}-1-4 n}{(t-s)} \frac{e^{-\frac{\left(y^{\prime}-1-4 n\right)^{2}}{4 \nu(t-s)}}}{\sqrt{4 \pi \nu(t-s)}} g_{\tau}^{+}\left(\xi^{\prime}, s\right)\right\|_{L^{2}\left(\xi^{\prime}\right)}^{2} \\
& \leq \sup _{0 \leq t \leq T}\left\|\int_{0}^{t} d s g_{\tau}^{+} \sum_{n=0}^{\infty} e^{-\left|\xi^{\prime}\right|(4 n+2)} \int_{-1}^{1} d y^{\prime} \frac{y^{\prime}-1-4 n}{(t-s)} \frac{\left.e^{-\left[\frac{y^{\prime}-1-4 n}{\sqrt{4 \nu(t-s)}}+\sqrt{\nu}\left|\xi^{\prime}\right|(t-s)^{1 / 2}\right.}\right]^{2}}{\sqrt{4 \pi \nu(t-s)}}\right\|^{2} \\
& +\sup _{0 \leq t \leq T}\left\|\int_{0}^{t} d s g_{\tau}^{+} \sum_{n=-\infty}^{-1} \int_{-1}^{1} d y^{\prime} e^{\left|\xi^{\prime}\right|\left(-2 y^{\prime}+4 n\right)} \frac{y^{\prime}-1-4 n}{(t-s)} \frac{\left.e^{-\left[\frac{y^{\prime}-1-4 n}{\sqrt{4 \nu(t-s)}}-\sqrt{\nu}\left|\xi^{\prime}\right|(t-s)^{1 / 2}\right.}\right]^{2}}{\sqrt{4 \pi \nu(t-s)}}\right\|^{2} \\
& \leq \sup _{0 \leq t \leq T}\left\|\int_{0}^{t} d s g_{\tau}^{+} \sum_{n=0}^{\infty} e^{-\left|\xi^{\prime}\right|(4 n+2)} \int_{-1}^{1} d y^{\prime} 2 \sqrt{\nu} \frac{d}{d y^{\prime}} \frac{\left.e^{-\left[\frac{y^{\prime}-1-4 n}{\sqrt{4 \nu(t-s)}}+\sqrt{\nu}\left|\xi^{\prime}\right|(t-s)^{1 / 2}\right.}\right]^{2}}{\sqrt{4 \pi(t-s)}}\right\|^{2} \\
& +\sup _{0 \leq t \leq T}\left\|\int_{0}^{t} d s g_{\tau}^{+} \sum_{n=0}^{\infty} e^{-\left|\xi^{\prime}\right|(4 n+2)} \int_{-1}^{1} d y^{\prime} 2 \sqrt{\nu}\left|\xi^{\prime}\right| \frac{\left.e^{-\left[\frac{y^{\prime}-1-4 n}{\sqrt{4 \nu(t-s)}}+\sqrt{\nu}\left|\xi^{\prime}\right|(t-s)^{1 / 2}\right.}\right]^{2}}{\sqrt{4 \pi(t-s)}}\right\|^{2} \\
& +\sup _{0 \leq t \leq T}\left\|\int_{0}^{t} d s g_{\tau}^{+} \sum_{n=-\infty}^{-1} \int_{-1}^{1} d y^{\prime} e^{\left|\xi^{\prime}\right|\left(-2 y^{\prime}+4 n\right)} 2 \sqrt{\nu} \frac{d}{d y^{\prime}} \frac{\left.e^{-\left[\frac{y^{\prime}-1-4 n}{\sqrt{4 \nu(t-s)}}-\sqrt{\nu}\left|\xi^{\prime}\right|(t-s)^{1 / 2}\right.}\right]^{2}}{\sqrt{4 \pi(t-s)}}\right\|^{2} \\
& +\sup _{0 \leq t \leq T}\left\|\int_{0}^{t} d s g_{\tau}^{+} \sum_{n=-\infty}^{-1} \int_{-1}^{1} d y^{\prime} e^{\left|\xi^{\prime}\right|\left(-2 y^{\prime}+4 n\right)} 2 \sqrt{\nu}\left|\xi^{\prime}\right| \frac{\left.e^{-\left[\frac{y^{\prime}-1-4 n}{\sqrt{4 \nu(t-s)}}-\sqrt{\nu}\left|\xi^{\prime}\right|(t-s)^{1 / 2}\right.}\right]^{2}}{\sqrt{4 \pi(t-s)}}\right\|^{2} \\
& =I_{1}+I_{2}+I_{3}+I_{4} \text {. }
\end{aligned}
$$

We now consider the four terms separately. We begin from $I_{1}$ :

$$
I_{1}=\sup _{0 \leq t \leq T} \| \int_{0}^{t} d s g_{\tau}^{+} \sqrt{\nu} \sum_{n=0}^{\infty} e^{-\left|\xi^{\prime}\right|(4 n+2)}
$$




$$
\begin{aligned}
& \times\left[\frac{e^{-\left[\frac{4 n}{\sqrt{4 \nu(t-s)}}-\sqrt{\nu}\left|\xi^{\prime}\right|(t-s)^{1 / 2}\right]^{2}}}{\sqrt{\pi(t-s)}}-\frac{\left.e^{-\left[\frac{4 n+2}{\sqrt{4 \nu(t-s)}}-\sqrt{\nu}\left|\xi^{\prime}\right|(t-s)^{1 / 2}\right.}\right]^{2}}{\sqrt{\pi(t-s)}} \|^{2}\right. \\
\leq & \sup _{0 \leq t \leq T}\left\|\int_{0}^{t} d s g_{\tau}^{+} 2 \sqrt{\nu} \sum_{n=0}^{\infty} e^{-\left|\xi^{\prime}\right|(4 n+2)} \frac{\left.e^{-\left[\frac{4 n}{\sqrt{4 \nu(t-s)}}-\sqrt{\nu}\left|\xi^{\prime}\right|(t-s)^{1 / 2}\right.}\right]^{2}}{\sqrt{\pi(t-s)}}\right\|^{2} \\
\leq & \sup _{0 \leq t \leq T}\left\|\int_{0}^{t} d s 2 \sqrt{\nu} e^{-\nu\left|\xi^{2}\right|(t-s)} e^{-2|\xi|} g_{\tau}^{+} \sum_{n=0}^{\infty} \frac{e^{-\frac{(4 n)^{2}}{4 \nu(t-s)}}}{\sqrt{\pi(t-s)}}\right\|^{2} \\
\leq & \sup _{0 \leq t \leq T}\left\|\int_{0}^{t} d s 4 \sqrt{\nu} \frac{1}{\sqrt{\pi(t-s)}} g_{\tau}^{+}\right\|^{2} \\
\leq & \frac{16 \nu T}{\pi} \sup _{0 \leq t \leq T}\left\|g_{\tau}^{+}\right\|^{2} .
\end{aligned}
$$

The estimate of the terms with the derivatives with respect to $x$ and $t$ is the same. Let us now pass to $I_{2}$. Introducing the variable $\eta=\left(y^{\prime}-1-4 n\right) / \sqrt{4 \nu(t-s)}+$ $\sqrt{\nu}|\xi|(t-s)^{1 / 2}$, we get

$$
\begin{aligned}
& I_{2} \\
& =\sup _{0 \leq t \leq T} \frac{1}{\pi}\left\|\int_{0}^{t} d s g_{\tau}^{+}\left(\xi^{\prime}, s\right) 4 \nu\left|\xi^{\prime}\right| \sum_{n=0}^{\infty} e^{-\left|\xi^{\prime}\right|(4 n+2)} \int_{\frac{-4 n-2}{\sqrt{4 \nu(t-s)}}+\sqrt{\nu}\left|\xi^{\prime}\right|(t-s)^{1 / 2}}^{\frac{-4 n}{\sqrt{4 \nu(t-s)}}+\sqrt{\nu}\left|\xi^{\prime}\right|(t-s)^{1 / 2}} d \eta e^{-\eta^{2}}\right\|^{2} \\
& \left.\leq \sup _{0 \leq t \leq T} \| \int_{0}^{t} d s g_{\tau}^{+} 8 \nu\left|\xi^{\prime}\right|\left(e^{-2|\xi|} \frac{1}{2} T \nu|\xi|+\sum_{n>\frac{1}{2} T \nu|\xi|} e^{-\left|\xi^{\prime}\right|(4 n+2)} e^{-\left[\frac{4 n}{\sqrt{4 \nu(t-s)}}-\sqrt{\nu}\left|\xi^{\prime}\right|(t-s)^{1 / 2}\right.}\right]\right) \|^{2} \\
& \leq\left(16 \nu^{4} T^{4}+4 \nu^{2} T^{2}\right) \sup _{0 \leq t \leq T}\left\|g_{\tau}^{+}\left(\xi^{\prime}, s\right)\right\|^{2} .
\end{aligned}
$$

The estimate of $I_{3}$ is analogous to the estimate of $I_{1}$, while the estimate of $I_{4}$ is analogous to the estimate of $I_{2}$. From the above estimates it is apparent that, choosing $T_{\alpha}$ small enough, one can make $\left\|\gamma^{-} U^{+} F_{1}\left(g_{\tau}^{+}, 0\right)\right\|_{l . T_{\alpha}}<\alpha\left|g_{\tau}^{+}\right|_{l, T}$ with $\alpha<1$.

Remark A.1. It is interesting to notice that, in the zero viscosity limit, the time $T_{\alpha}$ up to which the above estimate is valid grows to infinity: $T_{\alpha} \sim \nu^{-1}$.

Proof of Proposition 4.9. The trace at $y=-1$ of the operator $\mathcal{O}^{+}$is made of three terms: (1) the trace $\gamma^{-}$of $\mathcal{L}_{\tau}(\cdot, 0)$, which is estimated in Lemma 4.5 ; (2) the trace $\gamma^{-}$of $U^{+} F_{1}(\cdot, 0)$, which is estimated in Lemma 4.2; $(3)$ the trace $\gamma^{-}$of the operator $F_{1}(\cdot, 0)$, which can be estimated as follows:

$$
\begin{aligned}
& \sup _{0 \leq t \leq T}\left\|\gamma^{-} F_{1}\left(f_{\tau}^{+}, 0\right)\right\|_{L^{2}\left(\xi^{\prime}\right)}^{2} \\
& =\sup _{0 \leq t \leq T}\left\|\int_{0}^{t} d s e^{-\left[\nu \xi^{\prime 2}+i \xi^{\prime} U\right](t-s)} \sum_{n=-\infty}^{\infty} \frac{2+4 n}{\varepsilon(t-s)} \frac{e^{-\frac{(2+4 n)^{2}}{4 \nu(t-s)}}}{\sqrt{4 \pi(t-s)}} g_{\tau}^{+}\left(\xi^{\prime}, s\right)\right\|_{L^{2}\left(\xi^{\prime}\right)}^{2}
\end{aligned}
$$




$$
\begin{aligned}
& \leq \frac{4}{\pi} \sup _{0 \leq t \leq T}\left\|\int_{0}^{t} d s e^{-\nu \xi^{2}(t-s)} \sum_{n=0}^{\infty} \frac{1+2 n}{\varepsilon(t-s)} \frac{e^{-\frac{(1+2 n)^{2}}{\nu(t-s)}}}{\sqrt{(t-s)}} g_{\tau}^{+}\left(\xi^{\prime}, s\right)\right\|_{L^{2}\left(\xi^{\prime}\right)}^{2} \\
& \leq \frac{\nu}{\pi} \sup _{0 \leq t \leq T}\left\|\int_{0}^{t} d s \frac{e^{-\nu \xi^{\prime 2}(t-s)}}{(t-s)^{1 / 2}} g_{\tau}^{+}\left(\xi^{\prime}, s\right) \int_{0}^{\infty} d \eta \eta e^{-\eta^{2}}\right\|_{L^{2}\left(\xi^{\prime}\right)}^{2} \\
& \leq \frac{\nu T}{\pi} \sup _{0 \leq t \leq T}\left\|g_{\tau}^{+}\right\|_{L^{2}\left(\xi^{\prime}\right)}^{2} .
\end{aligned}
$$

As far as the term $\sup _{0 \leq t \leq T}\left\|\gamma^{-} U^{+}\left|\xi^{\prime}\right| F_{1}\left(f_{\tau}^{+}-\gamma^{+} \mathcal{L}_{\tau}\left(f_{N}^{+}, 0\right), 0\right)\right\|_{L^{2}\left(\xi^{\prime}\right)}^{2}$ is concerned, the estimate is analogous to the one given in the proof of Lemma 4.2. The only things one has to use are the fact that $\sqrt{\nu(t-s)}\left|\xi^{\prime}\right| e^{-\nu \xi^{\prime 2}(t-s)}$ is bounded and the regularizing property of the integration with respect to time of the factor $\frac{1}{\sqrt{t-s}}$. The estimate in (4.14) is thus achieved.

Proof of Proposition 4.10. The proof of Proposition 4.10 is analogous to the proof of Proposition 4.9.

Acknowledgments. The authors thank Professor Giga and an anonymous referee for the useful comments that helped to improve the paper and the presentation of the results.

\section{REFERENCES}

[1] M. Sammartino, The boundary layer analysis for Stokes equations on a half space, Comm. Partial Differential Equations, 22 (1997), pp. 749-771.

[2] M. Sammartino and R.E. Caflisch , Zero viscosity limit for analytic solutions of the NavierStokes equation on a half-space I. Existence for Euler and Prandtl equations, Comm. Math. Phys., 192 (1998), pp. 433-461.

[3] M. Sammartino AND R.E. CAflisch, Zero viscosity limit for analytic solutions of the NavierStokes equation on a half-space II. Construction of the Navier-Stokes solution, Comm. Math. Phys., 192 (1998), pp. 463-491.

[4] R. Temam and X. WAng, Asymptotic analysis of the linearized Navier-Stokes equations in a channel, Differential Integral Equations, 8 (1995), pp. 1591-1618.

[5] R. Temam And X. Wang, Asymptotic analysis of Oseen type equations in a channel at small viscosity, Indiana Univ. Math. J., 45 (1996), pp. 863-916.

[6] R. Temam and X. Wang, Boundary layers for Oseen's type equation in space dimension three, Russian J. Math. Phys., 5 (1998), pp. 227-246.

[7] S. UkaI, A solution formula for the Stokes equation in $\mathbb{R}_{+}^{n}$, Comm. Pure Appl. Math., 40 (1987), pp. 611-621.

[8] D.V. Widder, The Heat Equation, Academic Press, New York, 1975. 